\title{
Automatic settlement monitoring based on high-precision level
}

\author{
Xingang $\mathrm{Mou}^{1, \mathrm{a}}$, Jianxin Zhao ${ }^{1, \mathrm{b*}}$ and Xiao Zhou ${ }^{1, \mathrm{c}}$ \\ ${ }^{1}$ School of Mechanical and Electronic Engineering, Wuhan University of Technology, Wuhan, \\ China \\ amouxingang@163.com, ${ }^{\mathrm{b}} 1466765847 @ q q . c o m,{ }^{c} 17895099 @ q q . c o m$
}

Keywords: settlement monitoring; high precision level; automatic fault tolerance; encoding ruler identification

Abstract. This paper designs an automatic monitoring system of relative settlement of buildings, which mainly consists of three parts, that is automatic adjustment module, automatic fault tolerance correction code ruler module, automatic identification code ruler module. The monitoring system adopts the high precision level to measure the settlement distance. The code ruler has automatic fault tolerance correction function, which can reduce the interference of outside light effectively. Encoding ruler identification is optimized according to the design of the code ruler in this system. It has higher accuracy. With this design, experiment and analysis of the scheme, the feasibility of the scheme in this paper demonstrated the advantages of high-precision, lower cost, and less human investment.

\section{Introduction}

Automatic settlement monitoring developed rapidly in the past ten years. At present, the main companies of the automatic settlement monitoring market are the Swiss Leica, Japan Topcon and Sokkia and Zeiss of Germany. As the key technology of automatic settlement monitoring is the digital level, and the products of several major companies are different in encoding's standard, the principle of reading, so their main advantages and disadvantages are also roughly the same. Such as DINI12 $2^{[1]}$ of Germany's Zeiss, which has high measurement accuracy, high speed, high efficiency, but there are many sources of error. DINI12 is easily affected by electromagnetic field, CCD imaging quality, external light changes and other factors. DNA03 ${ }^{[2]}$ of Leica, SDL30 ${ }^{[3]}$ of Sokkia also have the advantages of high precision, strong objectivity and disadvantages of much interference, poor flexibility, environmental monitoring requirements.

In view of the shortcomings of the existing scheme, a kind of automatic subsidence monitoring system based on the precision level is designed in this paper.

\section{Overall scheme design of automatic settlement monitoring system}

In this paper, the design of automatic settlement monitoring system is mainly composed of a precision level, automatic leveling device, encoding scale, camera, PC, etc. Figure. 1 shows the system principle diagram.

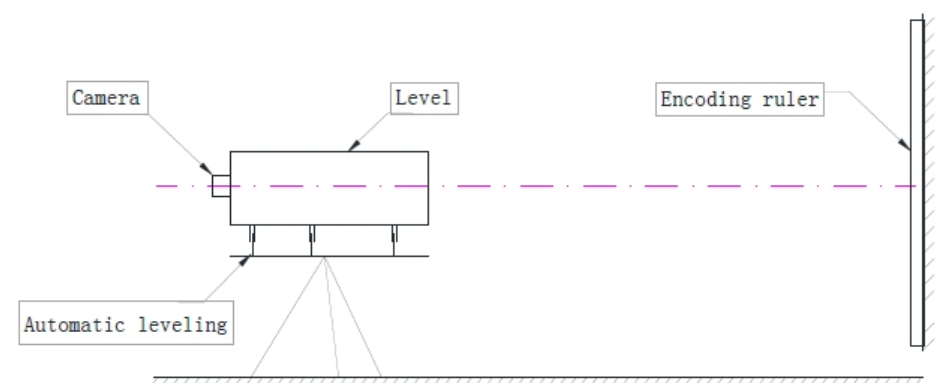

Figure.1 The system principle diagram

The system obtains the dip angle of $\mathrm{X}$ axis and $\mathrm{Y}$ axis that are belonged to platform by angle sensor measuring. Then the dip angle information is transferred into voltage signal, and the digital signal that the voltage signal is converted to through A/D conversion is transmitted to the microprocessor. Next the microprocessor converts the voltage signal into the dip angle. The feet of adjusting platform will 
move up and down by microprocessor adjusting steering gear turning when the dip angle is not 0 degrees. The block diagram of system is shown in figure. 2 . This system mainly includes three modules: automatic adjusting level system, encoding ruler, encoding ruler image processing and recognition.

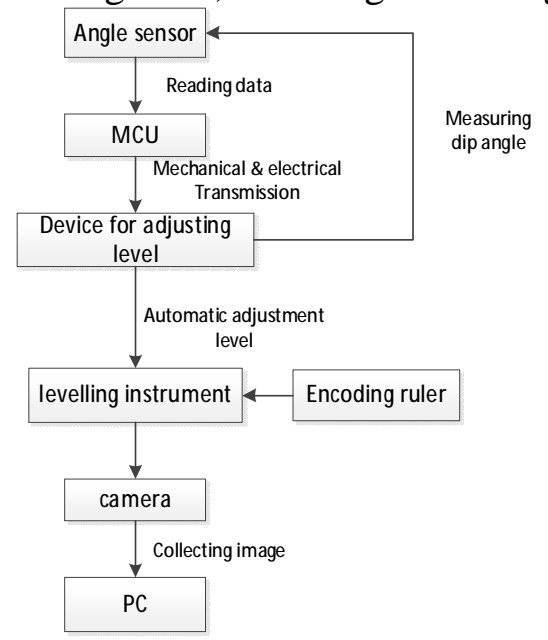

Figure.2 The block diagram of system

\section{Encoding ruler image processing and recognition}

\section{Design of precision level}

In this paper, the encoding ruler is a high precision, automatic, fault tolerance measuring scale. Each bar code of the ruler mainly consists of the black-and-white bar code, which according to the standard code range is not the same. And the number of single set bar code of black and white bar code is not the same. The total number of black and white bar code is $2(\mathrm{~N}+2)$ if the standard code range is $0 \sim 2^{\mathrm{N}}$. On the both sides of single set of bar code, two bar codes, consisting of black and white bar code, respectively are the beginning and the end bar code identification. The identification of the bar code is composed of multiple encodings which consist of a white bar code and a black bar code. The part of the encoding ruler in this paper is shown in Figure.3, the measurement range of the ruler is $0 \sim 45$ count.

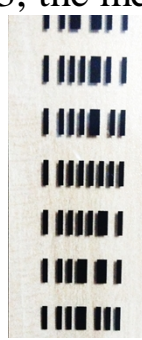

Figure. 3 Part of the encoding ruler

\section{Image processing}

Because the data of original image is too large, the edge information is not obvious, the noise is interfering and other issues, so the digital image is processed with gray transformation, filtering and noise reduction and edge detection before the bar code identification in this design.

In this paper, a new method is taken to realize image segmentation and the steps are shown as follows:

(1) After the image information is extracted from the edge information, the gray value of each row of the image is accumulated to the left.

(2) Obtain the mean of the sum result and keep it on the left side of the image.

(3) After all the pixels are processed, the median filtering is performed on result of the left side.

(4) Accumulate all the pixel values on the left, and then obtain the mean of the result as the threshold of the binaryzation processing. 
(5) According to the threshold of the left results for binarization, complete the binarization division of the right side of each line.

The result of the histogram segmentation is shown in figure. 4.

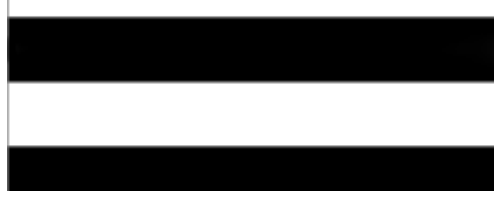

Figure. 4 Result of the histogram segmentation

\section{Encoding recognition}

The reading schematic of the bar code information is shown in the figure. 5. According to the bar code encoding rule, the two groups in the picture of the encoding information are 00000111 , 00000101 .

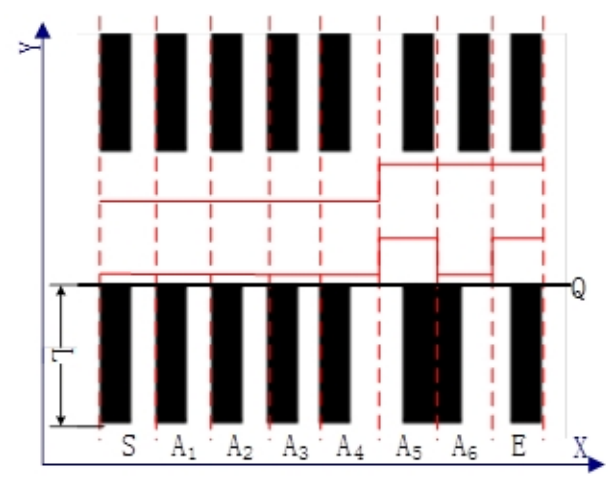

Figure. 5 Reading schematic of the bar code information

The beginning and the end of each group are respectively the start bit and stop bit. The transformation is starting at the first black bar code on the left and ending at the last black bar code on the right. If it is judged to be effective, convert the second sets of encoding which is closest to the line $\mathrm{Q}$ of the effective encoding in the image. Then compare the acquisition of the 8 bit encoding with the first conversion of encoding, if only one bit is different, the first encoding recognition is effective, and the results will be converted into normal decimal number $\mathrm{D}$ referring to encode table .

The decimal number obtained is regarded as the integer part. Then read the upper and lower pixels of the intersection of the valid bar code, and respectively record their vertical coordinates as a1, a2. The vertical coordinates of $\mathrm{Q}(\mathrm{a} 1)$, the upper edge of effective bar code ,is q. The center pixel vertical coordinate of the image is $\mathrm{h} / 2$. So the actual position, $\mathrm{Q}$ correspondence, is $\mathrm{M}$ :

$$
M=D+\frac{\frac{h}{2}-q}{a_{1}-a_{2}} \times L
$$

Then the mean value of the histogram elements is used as the threshold value. Process the image by the technology of vertical binarization segmentation, and obtain the encoding distribution of code on the encoding ruler. Finally, realize the recognition of $\mathrm{D}$ that is the integer part of encoding by reading out the solved encoding distribution. The solved encoding distribution of the code is shown in Figure. 6 . The part of black is the ground and the part of white is the effective bar code. The data, decoded out by procedures, is 00110001 that corresponding to the distance of ruler is $D=17 \mathrm{~cm}$.

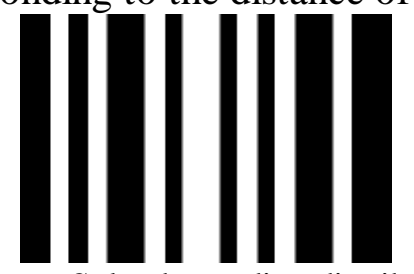

Figure. 6 Solved encoding distribution 


\section{Experimental results and analysis}

In order to better verify the accuracy and stability of the system, this paper has carried out several measurement experiments on the basis of the above. The code length of the bar code is $0.01 \mathrm{~mm}+5 \mathrm{~mm}$. The adjustment precision of lifting platform is $0.01 \mathrm{~mm}$. Since the lift range of the lifting platform is $0 \sim 10 \mathrm{~mm}$, therefore, the experimental lifting range is limited to $10 \mathrm{~mm}$ for guaranteeing the reliability of data, so the integer bar code has no reference function to our accuracy error.

The first set of data is the 10 set of data measured after the installation of the platform, the second set of data is to lift the table down after the measurement data $0.05 \mathrm{~mm}$.

From Table 1 it can be seen that the difference between the two groups of data is $0.0505 \mathrm{~mm}$ and the value of a minimum adjustment is $0.05 \mathrm{~mm}$ in practice. This has been in the adjustment of the instrument and equipment within the accuracy, so it can be basically considered that there is no error between the two groups.

Table 1 experimental data

\begin{tabular}{|c|l|l|l|l|}
\hline Group & First set & $a_{1}-a_{2}$ & Second set & $a_{1}-a_{2}$ \\
\hline 1 & 5.957 & 502 & 5.989 & 503 \\
\hline 2 & 5.984 & 503 & 6.075 & 503 \\
\hline 3 & 5.998 & 503 & 5.995 & 502 \\
\hline 4 & 5.987 & 503 & 6.052 & 502 \\
\hline 5 & 5.993 & 502 & 6.014 & 503 \\
\hline 6 & 5.937 & 501 & 6.003 & 503 \\
\hline 7 & 5.967 & 503 & 5.991 & 502 \\
\hline 8 & 5.983 & 503 & 5.995 & 503 \\
\hline 9 & 5.987 & 503 & 6.048 & 503 \\
\hline 10 & 5.898 & 503 & 6.034 & 503 \\
\hline Mean value & 5.9691 & 502.6 & 6.0196 & 502.7 \\
\hline
\end{tabular}

Figure. 7 is the measuring and operating interfaces of the first set data and the second set of data.

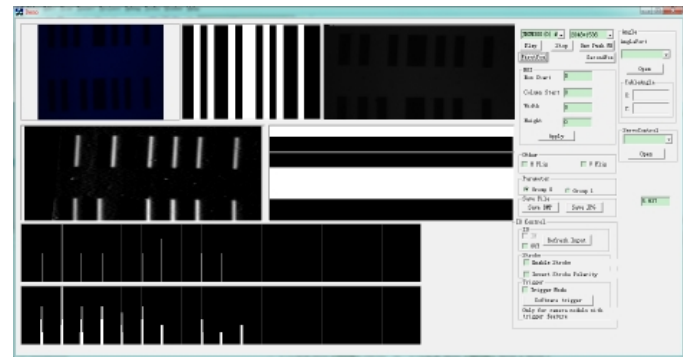

a. The first set data

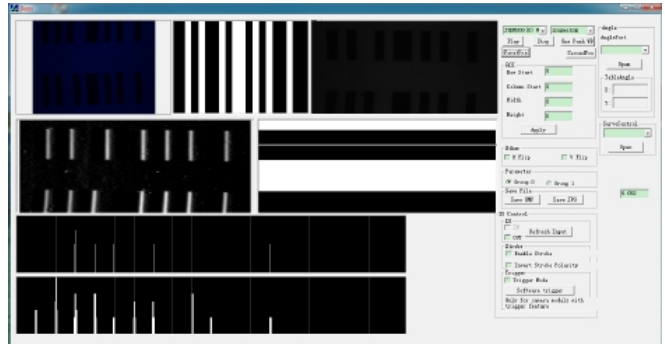

b. The second set of data

Figure. 7 Measuring and operating interfaces of the data

\section{Conclusion}

This paper designs an automatic subsidence monitoring system based on the precision level. The system can automatically complete the monitoring of the construction settlement with strong anti-interference ability, high accuracy, wide application range, can complete the monitoring of some complex environment.

\section{Acknowledgements}

This activity is thankfully supported by "the Fundamental Research Funds for the Central Universities(WUT: 2013-IV-090, 2012-IV-034)". 


\section{References}

[1] Liu Xuchun. Application of High Precision Digital Level in Sedimentation Observation[J]. Bulletin of Surveying, 2006, (1):58-61.

[2] Jin Weitao. Application of DNA03 digital levelling instrument in Yellow-River-Crossing works on mid route of South-to-North water transfer project[J]. Northwest Water Power, 2009, (2):15-17.

[3] Yuan Qingzhong, Li li. Suojia digital leveling instrument in settlement observation application[J]. Science \& Technology Vision, 2014, (27):110-110,271. 